\title{
An efficient synthesis of spiro- $\beta$-lactams having sulfenyl, sulfinyl and sulfonyl moiety
}

\author{
RESHMA $^{\mathrm{a}}$, RENU ARORA ${ }^{\mathrm{b}}$, GEETA HUNDAL $^{\mathrm{c}}$, AMAN BHALLA $^{\mathrm{d}}$ and S S BARI ${ }^{\mathrm{d}, *}$ \\ ${ }^{a}$ D. A. V. College, Sector 10, Chandigarh, India \\ ${ }^{\mathrm{b}}$ University Institute of Engineering and Technology, Panjab University, Chandigarh, India \\ ${ }^{\mathrm{c}}$ Department of Chemistry, Guru Nanak Dev University, Amritsar, India \\ ${ }^{\mathrm{d} D e p a r t m e n t}$ of Chemistry and Centre of Advanced studies in Chemistry, Panjab University, \\ Chandigarh 160014, India \\ e-mail: ssbari@pu.ac.in
}

MS received 9 June 2015; revised 8 August 2015; accepted 13 August 2015

\begin{abstract}
An efficient and a facile route to spiro- $\beta$-lactams is described. 3 -allyl-3-methylthio- $\beta$-lactams, which are synthesized through a Lewis acid mediated C-3 alkylation of the trans-3-chloro-3-methylthio- $\beta$ lactams, undergo a facile intrasulfenyl cyclization reaction in the presence of halogens like bromine and iodine to give spiro $\beta$-lactams in good yield. These halospiro- $\beta$-lactams are then subjected to dehalogenation reaction using Raney-nickel. The resulting spiro- $\beta$-lactams are further transformed into their sulfinyl and sulfonyl derivatives by using $m$-chloroperbenzoic acid as an oxidant.
\end{abstract}

Keywords. $\quad \beta$-lactams; intrasulfenyl cyclization; dehalogenation; sulfoxide; sulfone.

\section{Introduction}

The potential of $\beta$-lactams for the treatment of bacterial infection has been amply established. ${ }^{1,2}$ These miracle drugs have been the main contributors to the recent increase in human life expectancy. Therefore, large efforts are being made for the synthesis of $\beta$ lactam antibiotics with modified structures. However, the rapid emergence of bacterial strains resistant to most generally used members of this class of compounds requires a continuous effort towards the design and synthesis of novel derivatives that are stable to $\beta$-lactamases and possess high potency. In particular, spiro- $\beta$-lactams are interesting molecules because they may act as antiviral, ${ }^{3}$ antibacterial agents ${ }^{4}$ and also inhibit cholesterol absorption. ${ }^{5}$ They are also $\beta$ turn mimetics, ${ }^{6}$ with 4 -spiro- $\beta$-lactams in particular being synthetic precursors of cyclic $\alpha, \alpha$-disubstituted $\beta$-amino acids and peptide derivatives. ${ }^{7}$

Although a great deal of functionalized monocyclic $\beta$-lactams has been reported, relativly few reports have been devoted to the synthesis of spiro- $\beta$-lactams. ${ }^{8-11}$ Among the different strategies developed for the construction of $\beta$-lactams, ${ }^{12}$ the reaction of acid chlorides with imines i.e., Staudinger reaction ${ }^{13}$ constitutes one of the most popular procedures. In view of the ever growing applications of spiro- $\beta$-lactams, ranging

*For correspondence from their biological activity to their utility as synthetic intermediates in organic synthesis, we extended our work on C-3 alkylation of 3-methylthio- $\beta$-lactams to the synthesis of spiro- $\beta$-lactams. ${ }^{14}$ In previous work from our laboratory, we synthesized some spiro- $\beta$ lactams through halogen-mediated intrasulfenyl additions to alkynes/alkenes using cis-3-(prop-2-ynyoxy/enyloxy)- $\beta$-lactams as well as cis-3-allyl-3-benzylthio$\beta$-lactams. ${ }^{15,16}$ Continuing our studies as on the synthesis of spiro- $\beta$-lactams, it was envisaged to study the halocyclization reaction using cis-3-allyl-3-methylthio$\beta$-lactams also, which may prove to be an alternative substrate for the formation of C-3 spiro- $\beta$-lactams under very mild conditions. These studies were also aimed to find the effect of replacement of benzylthio by methylthio on epimeric ratio. Further, since a number of biologically significant molecules have sulfinyl and sulfonyl group, we extended these studies to the synthesis of spiro sulfinyl and spiro sulfonyl $\beta$-lactams.

\section{Experimental}

\subsection{General Information}

Melting points (M.p.) are uncorrected and are expressed in degree centigrade $\left({ }^{\circ} \mathrm{C}\right)$. Melting points were determined with a Thomas-Hoover capillary melting point apparatus. Infrared spectra (IR) were recorded using 
Perkin-Elmer Model 1430 spectrophotometer. Only principal absorption bands of interest are reported and expressed in $\mathrm{cm}^{-1} \cdot{ }^{1} \mathrm{H}$ NMR spectra were recorded using JEOL FT NMR System AL 300 (300 MHz) in the Department of Chemistry and Centre of Advance Studies in Chemistry and BRUKER AVANCE II 400 $(400 \mathrm{MHz})$ in the Sophisticated Analytical Instrumentation Facility (SAIF), Panjab University, Chandigarh. The chemical shift values are expressed in $\delta$ values (ppm) using tetramethylsilane (TMS) as internal standard. The coupling constant $J$ values are given in Hertz (Hz). While citing ${ }^{1} \mathrm{H}$ NMR data, following abbreviations have been used: s-singlet; br s-broad singlet; $d-$ doublet; t-triplet; q-quartet and m-multiplet. ${ }^{13} \mathrm{C} \mathrm{NMR}$, ${ }^{13} \mathrm{C}$ NMR DEPT 135 and DEPT 90; ${ }^{13} \mathrm{C}^{-1} \mathrm{H}$ Heteronuclear 2D Correlation Spectroscopy (COSY), HET$\mathrm{COR} ;{ }^{13} \mathrm{C}-{ }^{1} \mathrm{H}$ Heteronuclear single quantum correlation (HSQC); ${ }^{1} \mathrm{H}-{ }^{1} \mathrm{H}$ Homonuclear 2D Correlation Spectroscopy (COSY) were recorded using JEOL FT NMR System AL $300(300 \mathrm{MHz})$ and BRUKER AVANCE II 400 (400 MHz). In DEPT 135 (Distortionless Enhancement by Polarisation Transfer) spectra, suffix $(+)$ and $(-)$ signs means positive and negative amplitude of ${ }^{13} \mathrm{C}$ NMR resonances. Thin layer chromatography (TLC) was performed using TLC grade silica gel ' $G$ ' (Acme Synthetic Chemicals). The spots were made visible by exposing plates to iodine vapors. Column chromatography was performed with silica gel (Acme Synthetic Chemicals, 60-120 mesh) and eluted with ethyl acetate : hexanes mixtures unless otherwise stated. All commercially available compounds or reagents were used without further purification. All solvents used were of LR grade. Where necessary, the solvents were distilled and dried prior to use, when this seemed necessary by standard methods. Crystallographic data of compound 9a with CCDC no. 650921 in CIF format have been deposited with the Cambridge Crystallographic Data Centre. These data can be obtained free of charge from Cambridge Crystallographic Data Centre via www.ccdc.com.ac.uk/data_request/cif.

\subsection{General procedure for the halocyclization $(4,5,6,7)$}

To a stirred solution of cis-3-allyl-3-methylthio- $\beta$ lactam $3(1 \mathrm{mmol})$ in $5 \mathrm{~mL}$ of dry methylene chloride was added iodine/bromine $(1.2 \mathrm{mmol})$ at room temperature. The mixture was allowed to stir $(7-8 \mathrm{~h})$ at the same temperature. The progress of the reaction was monitored by TLC. After completion of the reaction, the reaction mixture was worked up according to the procedure as described in the cited reference. ${ }^{16}$ The spectroscopic data of compounds 4, 5 (a-c) and 6, 7 $(\mathbf{a}-\mathbf{b})$ are also reported in cited reference. ${ }^{16}$

2.2a 7-( $\alpha, \beta)$-Iodo-2-(4'-methylphenyl)-3-phenyl-thia2-aza-spiro[3.4]octan-1-one $(\mathbf{4 d}, \mathbf{5 d})$ : White solid; yield 69\%; IR $v_{\max }\left(\mathrm{CHCl}_{3}\right): 1743(\mathrm{C}=\mathrm{O}) \mathrm{cm}^{-1} ;{ }^{1} \mathrm{H}$ NMR $\left(\mathrm{CDCl}_{3}\right)$ (one isomer): $\delta 6.95-7.33(\mathrm{~m}, 9 \mathrm{H}, \mathrm{ArH})$, 4.97 (s, 1H, C4-H), 3.87-3.97 (m, 1H, CHI), 3.19-3.29 (m, 1H), 2.94-3.09 (m, 2H), 2.61-2.78 (m, 1H), 2.19 (s, $\left.3 \mathrm{H}, \mathrm{CH}_{3}\right) ;{ }^{1} \mathrm{H} \mathrm{NMR}\left(\mathrm{CDCl}_{3}\right)$ (other isomer): $\delta 6.95$ 7.33 (m, 9H, ArH), 5.07 (s, 1H, C4-H), 4.51-4.57 (m, 1H, CHI), 3.19-3.29 (m, 1H), 2.94-3.09 (m, 2H), 2.61$2.78(\mathrm{~m}, 1 \mathrm{H}), 2.19\left(\mathrm{~s}, 3 \mathrm{H}, \mathrm{CH}_{3}\right) ;{ }^{13} \mathrm{C} \mathrm{NMR}\left(\mathrm{CDCl}_{3}\right)$ (both isomers): $\delta$ 167.74, 165.97, 135.39, 135.08, $134.49,134.13,134.04,129.60,129.04,128.95$, $128.83,127.03,126.95,117.45,117.41,72.60,72.20$, $70.11,66.58,50.80,50.17,43.67,20.89,19.50,16.59$.

\subsection{General procedure for dehalogenation of $C-3$ spiro- $\beta$-lactams using Raney-nickel (8)}

The compounds 8 (a-c) were prepared by procedure as described in the cited reference. ${ }^{16}$ The spectroscopic data of compounds $\mathbf{8}(\mathbf{a}-\mathbf{b})$ are also reported in cited reference. $^{16}$

2.3a 2-(4'-Methoxyphenyl)-3-(4'-methoxyphenyl)-5thia-2-aza-spiro[3.4]octan-1-one (8c): White solid; yield $73 \%$; M.p. $155-156^{\circ} \mathrm{C}$; IR $v_{\max }\left(\mathrm{CHCl}_{3}\right): 1743$ $(\mathrm{C}=\mathrm{O}) \mathrm{cm}^{-1} ;{ }^{1} \mathrm{H}$ NMR $\left(\mathrm{CDCl}_{3}\right): \delta 6.74-7.25(\mathrm{~m}, 8 \mathrm{H}$, $\mathrm{ArH}), 4.95$ (s, 1H, C4-H), $3.78\left(\mathrm{~s}, 3 \mathrm{H}, \mathrm{OCH}_{3}\right), 3.72$ (s, 3H, OCH 3 ), 2.89-2.97 (1H, m, SCH $\left.\mathrm{CH}_{2} \mathrm{CH}_{\mathrm{a}} H_{b}\right)$, 2.75-2.83 (1H, m, $\left.\mathrm{SCH}_{2} \mathrm{CH}_{2} \mathrm{CH}_{a} \mathrm{H}_{\mathrm{b}}\right), 2.35-2.52(2 \mathrm{H}$, $\left.\mathrm{m}, \mathrm{SCH}_{2}\right), 2.24-2.34\left(\mathrm{~m}, 1 \mathrm{H}, \mathrm{SCH}_{2} \mathrm{CH}_{\mathrm{a}} H_{b}\right), 1.92-2.02$ $\left(\mathrm{m}, 1 \mathrm{H}, \mathrm{SCH}_{2} \mathrm{CH}_{a} \mathrm{H}_{\mathrm{b}}\right) ;{ }^{13} \mathrm{C} \mathrm{NMR}\left(\mathrm{CDCl}_{3}\right): \delta 168.36$, 159.73, 156.05, 130.97, 128.19, 128.06, 118.74,114.26, 114.17, 74.13, 68.32, 55.42, 55.18, 39.53, 34.02, 30.34; ${ }^{13} \mathrm{C}$ NMR (DEPT 135) $\left(\mathrm{CDCl}_{3}\right) \delta 128.19(+), 128.06$ $(+), 118.74(+), 114.26(+), 114.17(+), 68.32(+)$, $55.42(+), 55.18(+), 39.53(-), 34.02(-), 30.34(-)$; Analysis: calcd for $\mathrm{C}_{20} \mathrm{H}_{21} \mathrm{NO}_{3} \mathrm{~S}: \mathrm{C}, 67.58 ; \mathrm{H}, 5.95 ; \mathrm{N}$, 3.94\%; Found : C, 67.50; H, 5.90; N, 3.98\%.

$2.3 b \quad 2-(4$ '-Methylphenyl)-3-phenyl-5-thia-2-aza-spiro [3.4]octan-1-one $(\boldsymbol{8 d})$ : White solid; yield 70\%; M.p. 214-216 ${ }^{\circ}$; IR $v_{\max }\left(\mathrm{CHCl}_{3}\right): 1745(\mathrm{C}=\mathrm{O}) \mathrm{cm}^{-1} ;{ }^{1} \mathrm{H}$ NMR $\left(\mathrm{CDCl}_{3}\right): \delta$ 6.94-7.33 (m, 9H, ArH), $4.93(\mathrm{~s}$, $1 \mathrm{H}, \mathrm{C} 4-\mathrm{H}), 2.83-2.91\left(1 \mathrm{H}, \mathrm{m}, \mathrm{SCH}_{2} \mathrm{CH}_{2} \mathrm{CH}_{\mathrm{a}} H_{b}\right)$, 2.68-2.76 $\left(1 \mathrm{H}, \mathrm{m}, \mathrm{SCH}_{2} \mathrm{CH}_{2} \mathrm{CH}_{a} \mathrm{H}_{\mathrm{b}}\right), 2.29-2.48(2 \mathrm{H}$, $\mathrm{m}, \mathrm{SCH}_{2}$ ), 2.21-2.27 (m, 1H, $\left.\mathrm{SCH}_{2} \mathrm{CH}_{\mathrm{a}} H_{b}\right), 2.19$ (s, $\left.3 \mathrm{H}, \mathrm{CH}_{3}\right), 1.87-1.98\left(\mathrm{~m}, 1 \mathrm{H}, \mathrm{SCH}_{2} \mathrm{CH}_{a} \mathrm{H}_{\mathrm{b}}\right) ;{ }^{13} \mathrm{C} \mathrm{NMR}$ 
$\left(\mathrm{CDCl}_{3}\right): \delta 168.30,136.28,135.06,133.48,129.59$, 128.84, 128.64, 126.92, 117.40, 73.96, 68.48, 39.73, 33.93, 30.36, 21.02; Analysis: calcd for $\mathrm{C}_{19} \mathrm{H}_{19} \mathrm{NOS}$ : C, 73.75; H, 6.19; N, 4.53\%; Found : C, 73.65; H, 6.10; $\mathrm{N}, 4.59 \%$.

\subsection{General procedure for Synthesis of sulfoxide derivatives (9)}

To a solution of $8(1 \mathrm{mmol})$ in $\mathrm{CH}_{2} \mathrm{Cl}_{2}(2 \mathrm{~mL})$ at $0^{\circ} \mathrm{C}$ was added in one portion $m$-CPBA (' $1.2 \mathrm{mmol}$ ). The reaction mixture was stirred and monitored by TLC. After the completion of the reaction, the reaction mixture was poured into $5 \%$ aqueous $\mathrm{NaHSO}_{3}$ solution $(5 \mathrm{~mL})$. The aqueous layer was extracted with methylene chloride $(3 \times 10 \mathrm{~mL})$ and the combined organic layer was washed with brine $(10 \mathrm{~mL})$ and dried over anhydrous $\mathrm{Na}_{2} \mathrm{SO}_{4}$. The residue after solvent evaporation in vacuo, was purified by silica gel column chromatography using $15 \%$ ethyl acetate:hexanes as the eluent.

2.4a 2-(4'-Methoxyphenyl)-5-oxo-3-phenyl-5 $\lambda^{4}$-thia2-aza-spiro[3.4]octan-1-one (9a): Crystalline solid; yield $39 \%$; M.p. $144-145^{\circ} \mathrm{C}$; IR $v_{\max }\left(\mathrm{CHCl}_{3}\right)$ : $1743(\mathrm{C}=\mathrm{O}), 1048(\mathrm{~S}-\mathrm{O}) \mathrm{cm}^{-1} ;{ }^{1} \mathrm{H}$ NMR $\left(\mathrm{CDCl}_{3}\right)$ : $\delta$ 6.79-7.46 (m, 9H, ArH), $5.29(\mathrm{~s}, 1 \mathrm{H}, \mathrm{C} 4-\mathrm{H})$, $3.74\left(\mathrm{~s}, 3 \mathrm{H}, \mathrm{OCH}_{3}\right), 2.95-3.11\left(\mathrm{~m}, 3 \mathrm{H}, \mathrm{SOCH}_{2}\right.$, $\left.\mathrm{SOCH}_{2} \mathrm{CH}_{2} \mathrm{CH}_{\mathrm{a}} H_{\mathrm{b}}\right), 2.56-2.64\left(\mathrm{~m}, 2 \mathrm{H}, \mathrm{SOCH}_{2} \mathrm{CH}_{\mathrm{a}} H_{\mathrm{b}}\right.$, $\left.\mathrm{SOCH}_{2} \mathrm{CH}_{2} \mathrm{CH}_{\mathrm{a}} \mathrm{H}_{\mathrm{b}}\right), 2.44-2.49\left(\mathrm{~m}, 1 \mathrm{H}, \mathrm{SOCH}_{2} \mathrm{CH}_{\mathrm{a}}\right.$, $\left.\mathrm{H}_{\mathrm{b}}\right) ;{ }^{13} \mathrm{C} \mathrm{NMR}\left(\mathrm{CDCl}_{3}\right): \delta 162.31,156.61,133.22$, $130.18,129.50,129.19,127.07,118.79,114.53$, $85.63,61.75,55.51,51.85,28.56,22.83 ;{ }^{13} \mathrm{C}$ NMR (DEPT 135) $\left(\mathrm{CDCl}_{3}\right): \delta 129.46(+), 129.14(+)$, $127.02(+), 118.75(+), 114.48(+), 61.70(+), 55.46$ $(+), 51.80(-), 28.51(-), 22.78(-)$; Analysis: calcd for $\mathrm{C}_{19} \mathrm{H}_{19} \mathrm{NO}_{3} \mathrm{~S}: \mathrm{C}, 66.84 ; \mathrm{H}, 5.61 ; \mathrm{N}, 4.10 \%$; Found : C, 66.38; H, 5.29; N, 4.03\%. Crystal data for 9a: monoclinic, P 21/c 1, lattice parameters: $a-15.5520(10)$ $\AA ; b-10.3120(10) \AA ; c-10.3120(10) \AA ; \alpha-90^{\circ}$; $\beta-96.450(10)^{\circ} ; \gamma-90^{\circ}, V-1654.4(2) \AA^{3}, Z-4$, $D c=1.371 \mathrm{mg} / \mathrm{cm}^{3}, \mu(\mathrm{Mo}-\mathrm{K} \alpha)-0.213 \mathrm{~mm}^{-1}$, full matrix least-square on $\mathrm{F}^{2} ; R_{1}=0.0565, w R_{2}=0.1425$ for 3085 [R(int.) - 0.0361] observed reflections $[I>2 \sigma(I)]$ and $R_{1}-0.1039, w R_{2}-0.1760$ for all 3271 reflections.

$2.4 \mathrm{~b} \quad 2-\left(4^{\prime}-M e t h o x y p h e n y l\right)-5-o x o-3-p h e n y l-5 \lambda^{4}$-thia2-aza-spiro [3.4]octan-1-one (10a): Crystalline solid; yield 50\%; M.p. : $190-191^{\circ} \mathrm{C}$; IR $v_{\max }\left(\mathrm{CHCl}_{3}\right)$ : $1752(\mathrm{C}=\mathrm{O}), 1049(\mathrm{~S}-\mathrm{O}) \mathrm{cm}^{-1} ;{ }^{1} \mathrm{H}$ NMR $\left(\mathrm{CDCl}_{3}\right)$ : $\delta$ 6.78-7.40 (m, 9H, ArH), $5.03(\mathrm{~s}, 1 \mathrm{H}, \mathrm{C} 4-\mathrm{H})$, $3.74\left(\mathrm{~s}, 3 \mathrm{H}, \mathrm{OCH}_{3}\right), 2.89-2.97\left(\mathrm{~m}, 2 \mathrm{H}, \mathrm{SOCH}_{\mathrm{a}} H_{b}\right.$, $\left.\mathrm{SOCH}_{2} \mathrm{CH}_{2} \mathrm{CH}_{\mathrm{a}} H_{\mathrm{b}}\right), 2.78-2.85\left(\mathrm{~m}, 1 \mathrm{H}, \mathrm{SOCH}_{\mathrm{a}} \mathrm{H}_{\mathrm{b}}\right)$, 2.60-2.70 (m, 1H, $\left.\quad \mathrm{SOCH}_{2} \mathrm{CH}_{\mathrm{a}} H_{b}\right), \quad 2.37-2.44$ $\left(\mathrm{m}, \quad 1 \mathrm{H}, \quad \mathrm{SOCH}_{2} \mathrm{CH}_{2} \mathrm{CH}_{a} \mathrm{H}_{\mathrm{b}}\right), 2.04-2.16(\mathrm{~m}, 1 \mathrm{H}$, $\left.\mathrm{SOCH}_{2} \mathrm{CH}_{\mathrm{a}}, \mathrm{H}_{\mathrm{b}}\right) ;{ }^{13} \mathrm{C} \mathrm{NMR}\left(\mathrm{CDCl}_{3}\right): \delta 161.02,156.42$, $132.90,130.37,129.46,129.15,126.99,118.81$, 114.41, 83.75, 64.55, 55.46, 52.65, 30.82, 23.45, 30.82; ${ }^{13} \mathrm{C}$ NMR (DEPT 135) $\left(\mathrm{CDCl}_{3}\right): \delta 129.45(+), 129.15$ $(+), 126.98(+), 118.81(+), 114.40(+), 64.55(+)$, $55.46(+), 52.65(-), 30.82(-), 23.45(-)$.

2.4c 5-Oxo-2-phenyl-3-phenyl-5 $\lambda^{4}$-thia-2-aza-spiro [3.4]octan-1-one (9b): Crystalline solid; yield 41\%; M.p. ${ }^{175}-177^{\circ} \mathrm{C}$; IR $v_{\max }\left(\mathrm{CHCl}_{3}\right): 1749(\mathrm{C}=\mathrm{O})$, $1049 \mathrm{~cm}^{-1} ;{ }^{1} \mathrm{H}$ NMR $\left(\mathrm{CDCl}_{3}\right): \delta \quad 7.08-7.47 \quad(\mathrm{~m}$, $10 \mathrm{H}, \mathrm{ArH}), 5.28(\mathrm{~s}, 1 \mathrm{H}, \mathrm{C} 4-\mathrm{H}), 2.96-3.14(\mathrm{~m}, 3 \mathrm{H}$, $\left.\mathrm{SOCH}_{2}, \quad \mathrm{SOCH}_{2} \mathrm{CH}_{2} \mathrm{CH}_{\mathrm{a}} H_{b}\right), 2.58-2.68(\mathrm{~m}, 2 \mathrm{H}$, $\left.\mathrm{SOCH}_{2} \mathrm{CH}_{\mathrm{a}} H_{b}, \mathrm{SOCH}_{2} \mathrm{CH}_{2} \mathrm{CH}_{a} \mathrm{H}_{\mathrm{b}}\right), 2.44-2.52$ (m, $\left.1 \mathrm{H}, \mathrm{SOCH}_{2} \mathrm{CH}_{a} \mathrm{H}_{\mathrm{b}}\right) ;{ }^{13} \mathrm{C} \mathrm{NMR}\left(\mathrm{CDCl}_{3}\right): \delta 162.97$, $136.71,133.13,129.57,129.35,129.25,127.04,124.81$, $117.49,85.61,61.74,51.91,28.66,22.88 ;{ }^{13} \mathrm{C}$ NMR (DEPT 135) $\left(\mathrm{CDCl}_{3}\right): \delta 129.50(+), 129.28(+)$, $129.18(+), 126.97(+), 124.74(+), 117.41(+), 61.66$ $(+), 51.84(-), 28.58(-), 22.80(-)$.

2.4d 5-Oxo-2-phenyl-3-phenyl-5 $\lambda^{4}$-thia-2-aza-spiro [3.4]octan-1-one (10b): Crystalline solid; yield $47 \%$; M.p. $234-235^{\circ} \mathrm{C}$; IR $v_{\max }\left(\mathrm{CHCl}_{3}\right): 1756$ $(\mathrm{C}=\mathrm{O}), 1050 \quad(\mathrm{~S}-\mathrm{O}) \mathrm{cm}^{-1} ;{ }^{1} \mathrm{H}$ NMR $\left(\mathrm{CDCl}_{3}\right): \delta$ 6.98-7.37 (m, 10H, ArH), $4.99(\mathrm{~s}, 1 \mathrm{H}, \mathrm{C} 4-\mathrm{H})$, 2.71-2.92 (m, 3H, $\left.\mathrm{SOCH}_{2}, \quad \mathrm{SOCH}_{2} \mathrm{CH}_{2} \mathrm{CH}_{\mathrm{a}} \mathrm{H}_{b}\right)$, 2.55-2.62 (m, $\left.\quad 1 \mathrm{H}, \quad \mathrm{SOCH}_{2} \mathrm{CH}_{\mathrm{a}} H_{b}\right), \quad 2.29-2.38$ $\left(\mathrm{m}, \quad 1 \mathrm{H}, \quad \mathrm{SOCH}_{2} \mathrm{CH}_{2} \mathrm{CH}_{a} \mathrm{H}_{\mathrm{b}}\right), \quad 2.02-2.09(\mathrm{~m}, \quad 1 \mathrm{H}$, $\left.\mathrm{SOCH}_{2} \mathrm{CH}_{a} \mathrm{H}_{\mathrm{b}}\right) ;{ }^{13} \mathrm{C} \mathrm{NMR}\left(\mathrm{CDCl}_{3}\right): \delta 161.53,136.81$, 132.79, 129.46, 129.15, 126.89, 124.48, 117.45, 83.70, 64.46, 52.60, 30.83, 23.38; ${ }^{13} \mathrm{C}$ NMR (DEPT 135) $\left(\mathrm{CDCl}_{3}\right): \delta 129.46(+), 129.15(+), 126.89(+), 124.48$ $(+), 117.45(+), 64.46(+), 52.60(-), 30.83(-)$, $23.38(-)$.

2.4e 2-(4'-Methoxyphenyl)-3-(4'-methoxyphenyl)-5oxo-5 $\lambda^{4}$-thia-2-aza-spiro[3.4]octan-1-one (9c): Crystalline solid; yield 47\%; M.p. $199-200^{\circ} \mathrm{C}$; IR $v_{\max }$ $\left(\mathrm{CHCl}_{3}\right): 1742(\mathrm{C}=\mathrm{O}), 1047(\mathrm{~S}-\mathrm{O}) \mathrm{cm}^{-1} ;{ }^{1} \mathrm{H}$ NMR $\left(\mathrm{CDCl}_{3}\right): \delta$ 6.70-7.29 (m, 8H, ArH), $5.11(\mathrm{~s}, 1 \mathrm{H}$, $\mathrm{C} 4-\mathrm{H}), 3.72$ (s, 3H, $\left.\mathrm{OCH}_{3}\right), 3.67\left(\mathrm{~s}, 3 \mathrm{H}, \mathrm{OCH}_{3}\right), 2.85-$ $3.07\left(\mathrm{~m}, 3 \mathrm{H}, \mathrm{SOCH}_{2}, \mathrm{SOCH}_{2} \mathrm{CH}_{2} \mathrm{CH}_{\mathrm{a}} \mathrm{H}_{b}\right), 2.34-2.60$ (m, $3 \mathrm{H}, \mathrm{SOCH}_{2} \mathrm{CH}_{2}, \mathrm{SOCH}_{2} \mathrm{CH}_{2} \mathrm{CH}_{a} \mathrm{H}_{\mathrm{b}}$ ); ${ }^{13} \mathrm{C} \mathrm{NMR}$ $\left(\mathrm{CDCl}_{3}\right): \delta 162.24,160.24,156.49,130.18,128.23$, 124.98, 118.73, 114.54, 114.43, 85.72, 61.35, 55.36, 55.12, 51.69, 28.44, 22.78; ${ }^{13} \mathrm{C}$ NMR (DEPT 135) 
$\left(\mathrm{CDCl}_{3}\right): \delta 128.23(+), 118.73(+), 114.54(+), 114.43$ $(+), 61.35(+), 55.36(+), 55.12(+), 51.69(-), 28.44$ $(-), 22.78(-)$.

$2.4 \mathrm{f}$ 2-(4'-Methoxyphenyl)-3-(4'-methoxyphenyl)-5oxo-5 $\lambda^{4}$-thia-2-aza-spiro[3.4]octan-1-one $(10 c)$ : Crystalline solid; yield $42 \%$; M.p. $169-170^{\circ} \mathrm{C}$; IR $v_{\max }\left(\mathrm{CHCl}_{3}\right): 1751(\mathrm{C}=\mathrm{O}), 1038(\mathrm{~S}-\mathrm{O}) \mathrm{cm}^{-1} ;{ }^{1} \mathrm{H}$ NMR $\left(\mathrm{CDCl}_{3}\right): \delta$ 6.68-7.26 (m, 8H, ArH), $4.89(\mathrm{~s}$, $1 \mathrm{H}, \mathrm{C} 4-\mathrm{H}), 3.74\left(\mathrm{~s}, 3 \mathrm{H}, \mathrm{OCH}_{3}\right), 3.66\left(\mathrm{~s}, 3 \mathrm{H}, \mathrm{OCH}_{3}\right)$, 2.70-2.91 (m, 3H, $\left.\quad \mathrm{SOCH}_{2}, \quad \mathrm{SOCH}_{2} \mathrm{CH}_{2} \mathrm{CH}_{\mathrm{a}} H_{b}\right)$, 2.52-2.61 (m, $\left.\quad 1 \mathrm{H}, \quad \mathrm{SOCH}_{2} \mathrm{CH}_{\mathrm{a}} H_{b}\right), \quad 2.25-2.34$ $\left(\mathrm{m}, \quad 1 \mathrm{H}, \quad \mathrm{SOCH}_{2} \mathrm{CH}_{2} \mathrm{CH}_{a} \mathrm{H}_{\mathrm{b}}\right), \quad 1.94-2.05(\mathrm{~m}, \quad 1 \mathrm{H}$, $\left.\mathrm{SOCH}_{2} \mathrm{CH}_{a} \mathrm{H}_{\mathrm{b}}\right) ;{ }^{13} \mathrm{C} \mathrm{NMR}\left(\mathrm{CDCl}_{3}\right): \delta 160.99,160.30$, $156.33,130.38,128.35,124.55,118.77,114.43$, 114.33, 83.79, 64.18, 55.35, 55.22, 52.35, 30.52, 23.18; ${ }^{13} \mathrm{C}$ NMR (DEPT 135) $\left(\mathrm{CDCl}_{3}\right): \delta 128.35(+), 118.77$ $(+), 114.43(+), 114.33(+), 64.18(+), 55.35(+)$, $55.25(+), 52.35(-), 30.52(-), 23.18(-)$.

2.4g 2-(4'-Methylphenyl)-5-oxo-3-phenyl-5 $\lambda^{4}$-thia-2aza-spiro[3.4]octan-1-one (9d): Crystalline solid; yield 33\%; IR $v_{\max }\left(\mathrm{CHCl}_{3}\right): 1745(\mathrm{C}=\mathrm{O}), 1049$ (S-O) $\mathrm{cm}^{-1} ;{ }^{1} \mathrm{H}$ NMR $\left(\mathrm{CDCl}_{3}\right): \delta$ 7.06-7.46 (m, 9H, ArH) $5.25(\mathrm{~s}, 1 \mathrm{H}, \mathrm{C} 4-\mathrm{H}), 2.95-3.10(\mathrm{~m}, 3 \mathrm{H}$, $\left.\mathrm{SOCH}_{2}, \quad \mathrm{SOCH}_{2} \mathrm{CH}_{2} \mathrm{CH}_{\mathrm{a}} \mathrm{H}_{b}\right), 2.57-2.63(\mathrm{~m}, \quad 2 \mathrm{H}$, $\mathrm{SOCH}_{2} \mathrm{CH}_{\mathrm{a}} H_{b}$, $\left.\mathrm{SOCH}_{2} \mathrm{CH}_{2} \mathrm{CH}_{a} \mathrm{H}_{\mathrm{b}}\right)$, 2.46-2.49 (m, $\left.1 \mathrm{H}, \mathrm{SOCH}_{2} \mathrm{CH}_{a} \mathrm{H}_{\mathrm{b}}\right), 2.28\left(\mathrm{~s}, 3 \mathrm{H}, \mathrm{CH}_{3}\right)$.

2.4h 2-(4'-Methylphenyl)-5-oxo-3-phenyl-5 $\lambda^{4}$-thia-2aza-spiro[3.4]octan-1-one (10d): Crystalline solid; yield 40\%; M.p. $132-134^{\circ} \mathrm{C}$; IR $v_{\max }\left(\mathrm{CHCl}_{3}\right)$ : $1755(\mathrm{C}=\mathrm{O}), 1055(\mathrm{~S}-\mathrm{O}) \mathrm{cm}^{-1} ;{ }^{1} \mathrm{H}$ NMR $\left(\mathrm{CDCl}_{3}\right)$ : $\delta$ 6.97-7.35 (m, 9H, ArH), $4.96(\mathrm{~s}, 1 \mathrm{H}, \mathrm{C} 4-\mathrm{H})$, 2.71-2.92 (m, 3H, $\left.\quad \mathrm{SOCH}_{2}, \quad \mathrm{SOCH}_{2} \mathrm{CH}_{2} \mathrm{CH}_{\mathrm{a}} \mathrm{H}_{b}\right)$, 2.55-2.66 (m, 1H, $\left.\quad \mathrm{SOCH}_{2} \mathrm{CH}_{\mathrm{a}} H_{b}\right), \quad 2.27-2.36$ $\left(\mathrm{m}, \quad 1 \mathrm{H}, \quad \mathrm{SOCH}_{2} \mathrm{CH}_{2} \mathrm{CH}_{a} \mathrm{H}_{\mathrm{b}}\right), \quad 1.97-2.10 \quad(\mathrm{~m}, \quad 1 \mathrm{H}$, $\left.\mathrm{SOCH}_{2} \mathrm{CH}_{a} \mathrm{H}_{\mathrm{b}}\right), 2.21\left(\mathrm{~s}, 3 \mathrm{H}, \mathrm{CH}_{3}\right) ;{ }^{13} \mathrm{C} \mathrm{NMR}\left(\mathrm{CDCl}_{3}\right)$ : $\delta$ 174.08, 151.53, 132.97, 129.67, 129.43, 129.15, $126.96,117.41,64.44,54.64,52.53,30.79,23.33$, 20.95; ${ }^{13} \mathrm{C}$ NMR (DEPT 135) $\left(\mathrm{CDCl}_{3}\right): \delta 129.68(+)$, $129.44(+), 129.15(+), 126.93(+), 117.41(+), 64.45$ $(+), 52.64(-), 30.87(-), 23.45(-), 20.95(+)$.

\subsection{General procedure for Synthesis of sulfone} derivatives (11)

To a solution of $8(1 \mathrm{mmol})$ in $\mathrm{CH}_{2} \mathrm{Cl}_{2}(2 \mathrm{~mL})$ was added in one portion $m$-CPBA $(2 \mathrm{mmol})$ at room temperature. The reaction mixture was stirred and monitored by TLC. After the completion of the reaction, the reaction mixture was poured into $5 \%$ aqueous $\mathrm{NaHSO}_{3}$ solution $(5 \mathrm{~mL})$. The aqueous layer was extracted with methylene chloride $(3 \times 10 \mathrm{~mL})$ and the combined organic layer was washed with brine $(10 \mathrm{~mL})$ and dried over anhydrous $\mathrm{Na}_{2} \mathrm{SO}_{4}$. The residue after solvent evaporation in vacuo, was purified by silica gel column chromatography using $15 \%$ ethyl acetate:hexanes as the eluent.

2.5a 2-(4'-methoxyphenyl)-5,5-dioxo-3-phenyl-5 $\lambda^{6}$ thia-2-aza-spiro[3.4]octan-1-one (11a): Crystalline solid; yield 68\%; IR $v_{\max }\left(\mathrm{CHCl}_{3}\right): 1734(\mathrm{C}=\mathrm{O}), 1394$, $1110(\mathrm{~S}=\mathrm{O}) \mathrm{cm}^{-1} ;{ }^{1} \mathrm{H}$ NMR $\left(\mathrm{CDCl}_{3}\right): \delta 6.70-7.42(\mathrm{~m}$, $9 \mathrm{H}, \mathrm{ArH}), 5.14(\mathrm{~s}, 1 \mathrm{H}, \mathrm{C} 4-\mathrm{H}), 3.67\left(\mathrm{~s}, 3 \mathrm{H}, \mathrm{OCH}_{3}\right)$, 3.21-3.31 (m, $\left.1 \mathrm{H}, \mathrm{SO}_{2} \mathrm{CH}_{\mathrm{a}} H_{b}\right), 2.90-3.00(\mathrm{~m}, 1 \mathrm{H}$, $\left.\mathrm{SO}_{2} \mathrm{CH}_{a} \mathrm{H}_{\mathrm{b}}\right), 2.59-2.65\left(\mathrm{~m}, 2 \mathrm{H}, \mathrm{SO}_{2} \mathrm{CH}_{2} \mathrm{CH}_{2} \mathrm{CH}_{2}\right)$, 2.33-2.41 (m, $\left.1 \mathrm{H}, \mathrm{SO}_{2} \mathrm{CH}_{2} \mathrm{CH}_{\mathrm{a}} H_{b}\right), 2.02-2.18(\mathrm{~m}, 1 \mathrm{H}$, $\left.\mathrm{SO}_{2} \mathrm{CH}_{2} \mathrm{CH}_{a} \mathrm{H}_{\mathrm{b}}\right) ;{ }^{13} \mathrm{C}$ NMR $\left(\mathrm{CDCl}_{3}\right): \delta 160.71,156.61$, $130.53,129.96,129.58,128.63,127.79,118.88$, $114.41,76.81,62.56,55.41,50.63,28.38,16.45 ;{ }^{13} \mathrm{C}$ NMR (DEPT 135) $\left(\mathrm{CDCl}_{3}\right): \delta 129.58(+), 128.63(+)$, $127.79(+), 118.88(+), 114.41(+), 62.56(+), 55.41$ $(+), 50.63(-), 28.38(-), 16.45(-)$.

2.5b 5,5-Dioxo-2-phenyl-3-phenyl-5 $\lambda^{6}$-thia-2-azaspiro[3.4]octan-1-one (11b): White solid; yield $71 \%$; IR $v_{\max }\left(\mathrm{CHCl}_{3}\right): 1751(\mathrm{C}=\mathrm{O}), 1323,1132$ $(\mathrm{S}=\mathrm{O}) \mathrm{cm}^{-1} ;{ }^{1} \mathrm{H}$ NMR $\left(\mathrm{CDCl}_{3}\right): \delta$ 7.09-7.50 $(\mathrm{m}$, $10 \mathrm{H}, \mathrm{ArH}), 5.25(\mathrm{~s}, 1 \mathrm{H}, \mathrm{C} 4-\mathrm{H}), 3.30-3.38(\mathrm{~m}, 1 \mathrm{H}$, $\left.\mathrm{SO}_{2} \mathrm{CH}_{\mathrm{a}} H_{b}\right), 3.00-3.07\left(\mathrm{~m}, 1 \mathrm{H}, \mathrm{SO}_{2} \mathrm{CH}_{a} \mathrm{H}_{\mathrm{b}}\right), 2.68$ $2.72\left(\mathrm{~m}, 2 \mathrm{H}, \mathrm{SO}_{2} \mathrm{CH}_{2} \mathrm{CH}_{2} \mathrm{CH}_{2}\right), 2.43-2.48(\mathrm{~m}, 1 \mathrm{H}$, $\left.\mathrm{SO}_{2} \mathrm{CH}_{2} \mathrm{CH}_{\mathrm{a}} H_{b}\right), 2.19-2.26\left(\mathrm{~m}, 1 \mathrm{H}, \mathrm{SO}_{2} \mathrm{CH}_{2} \mathrm{CH}_{a} \mathrm{H}_{\mathrm{b}}\right.$ ); ${ }^{13} \mathrm{C} \mathrm{NMR}\left(\mathrm{CDCl}_{3}\right): \delta 161.38,136.56,130.48,129.73$, 129.31, 128.77, 127.83, 124.93, 117.62, 81.35, 62.59, $50.75,28.54,16.56 ;{ }^{13} \mathrm{C}$ NMR (DEPT 135) $\left(\mathrm{CDCl}_{3}\right)$ : $\delta 129.67(+), 129.25(+), 128.72(+), 127.77(+)$, $124.87(+), 117.56(+), 62.53(+), 50.69(-), 28.49$ $(-), 16.50(-)$.

2.5c 2-(4'-Methoxyphenyl)-3-(4'-methoxyphenyl)-5, 5-dioxo-5 $\lambda^{6}$-thia-2-aza-spiro[3.4]octan-1-one (11c): White solid; yield 76\%; IR $\left(\mathrm{CHCl}_{3}\right)$ : $1752(\mathrm{C}=\mathrm{O})$, 1311, $1135(\mathrm{~S}=\mathrm{O}) \mathrm{cm}^{-1} ;{ }^{1} \mathrm{H}$ NMR $\left(\mathrm{CDCl}_{3}\right): \delta 6.78-$ $7.41(\mathrm{~m}, 8 \mathrm{H}, \operatorname{ArH}), 5.17(\mathrm{~s}, 1 \mathrm{H}, \mathrm{C} 4-\mathrm{H}), 3.79(\mathrm{~s}$, $\left.3 \mathrm{H}, \mathrm{OCH}_{3}\right), 3.74\left(\mathrm{~s}, 3 \mathrm{H}, \mathrm{OCH}_{3}\right), 3.29-3.37(\mathrm{~m}, 1 \mathrm{H}$, $\left.\mathrm{SO}_{2} \mathrm{CH}_{\mathrm{a}} H_{\mathrm{b}}\right), 3.00-3.07\left(\mathrm{~m}, 1 \mathrm{H}, \quad \mathrm{SO}_{2} \mathrm{CH}_{\mathrm{a}} \mathrm{H}_{\mathrm{b}}\right), 2.65-$ $2.69\left(\mathrm{~m}, 2 \mathrm{H}, \mathrm{SO}_{2} \mathrm{CH}_{2} \mathrm{CH}_{2} \mathrm{CH}_{2}\right), 2.41-2.46(\mathrm{~m}, 1 \mathrm{H}$, $\left.\mathrm{SO}_{2} \mathrm{CH}_{2} \mathrm{CH}_{\mathrm{a}} H_{\mathrm{b}}\right), 2.21-2.24\left(\mathrm{~m}, 1 \mathrm{H}, \mathrm{SO}_{2} \mathrm{CH}_{2} \mathrm{CH}_{\mathrm{a}} \mathrm{H}_{\mathrm{b}}\right)$; ${ }^{13} \mathrm{C}$ NMR $\left(\mathrm{CDCl}_{3}\right): \delta 160.77,160.41,156.56,130.01$, 129.09, 122.32, 118.92, 114.39, 114.07, 76.75, 62.27, $55.41,50.59,28.25,16.40 ;{ }^{13} \mathrm{C}$ NMR (DEPT 135) 
$\left(\mathrm{CDCl}_{3}\right): \delta 129.09(+), 118.92(+), 114.39(+), 114.07$ $(+), 62.27(+), 55.41(+), 55.13(+), 50.59(-), 28.25$ $(-), 16.40(-)$.

2.5d 2-(4'-Methylphenyl)-5,5-dioxo-3-phenyl-5 $\lambda^{6}$ thia-2-aza-spiro[3.4]octan-1-one (11d): White solid; yield 66\%; IR $v_{\max }\left(\mathrm{CHCl}_{3}\right): 1752(\mathrm{C}=\mathrm{O}), 1321$, $1132(\mathrm{~S}=\mathrm{O}) \mathrm{cm}^{-1} ;{ }^{1} \mathrm{H}$ NMR $\left(\mathrm{CDCl}_{3}\right): \delta$ 7.05-7.49 (m, 9H, ArH), 5.22 (s, 1H, C4-H), 3.30-3.37 (m, 1H, $\left.\mathrm{SO}_{2} \mathrm{CH}_{\mathrm{a}} H_{b}\right), 2.99-3.06\left(\mathrm{~m}, 1 \mathrm{H}, \mathrm{SO}_{2} \mathrm{CH}_{a} \mathrm{H}_{\mathrm{b}}\right), 2.67-$ $2.71\left(\mathrm{~m}, 2 \mathrm{H}, \mathrm{SO}_{2} \mathrm{CH}_{2} \mathrm{CH}_{2} \mathrm{CH}_{2}\right), 2.41-2.48(\mathrm{~m}, 1 \mathrm{H}$, $\left.\mathrm{SO}_{2} \mathrm{CH}_{2} \mathrm{CH}_{\mathrm{a}} \mathrm{H}_{b}\right), 2.27\left(\mathrm{~s}, 3 \mathrm{H}, \mathrm{CH}_{3}\right), 2.18-2.26(\mathrm{~m}$, $\left.1 \mathrm{H}, \mathrm{SO}_{2} \mathrm{CH}_{2} \mathrm{CH}_{a} \mathrm{H}_{\mathrm{b}}\right) ;{ }^{13} \mathrm{C} \mathrm{NMR}\left(\mathrm{CDCl}_{3}\right): \delta 161.09$, $134.66,134.13,130.60,129.79,129.66,128.73$, $127.84,117.54,62.51,50.72,28.51,21.02,16.55 ;{ }^{13} \mathrm{C}$ NMR (DEPT 135) $\left(\mathrm{CDCl}_{3}\right): \delta 129.73(+), 129.61(+)$, $128.68(+), 127.79(+), 117.49(+), 62.45(+), 50.67$ $(-), 28.45(-), 20.97(+), 16.49(-)$.

\section{Results and Discussion}

The $\beta$-lactams 1a-d required for this study were prepared from methylthioacetic acid and the appropriate Schiff's bases by the reported procedure. ${ }^{14}$ Furthermore, the trans-3-methylthio-3-chloroazetidin-2one 2a-d were obtained by the stereospecific chlorination of 1a-d, using N-chlorosuccinimide (NCS) with catalytic amount of AIBN in refluxing carbon tetrachloride by the reported procedure. ${ }^{14}$ These on treatment with trimethyl allylsilane and Lewis acid such as $\mathrm{SnCl}_{4}$ in dry dichloromethane under nitrogen atmosphere at $0^{\circ} \mathrm{C}$ to $-5^{\circ} \mathrm{C}$ afforded cis-3-allyl-3-methylthioazetidin2 -ones 3 by the reported procedure. ${ }^{14}$ For the synthesis of various C-3 spiro- $\beta$-lactams, cis-3-allyl-3methylthio- $\beta$-lactams 3 were considered as the most suitable substrates capable of undergoing halogenmediated intrasulfenyl cyclization reaction (schemes 1 and 2, table 1).

The halocyclization studies were initially carried out using $\beta$-lactams 3a with iodine (1 equiv) in dry dichloromethane at room temperature. The TLC profile showed appearance of two very close spots having $\mathrm{R}_{\mathrm{f}}$ slightly lower than the starting substrate. These two spots correspond to $7 \alpha$-iodo-2(4'-methoxyphenyl)-3-phenyl-5-thia-2-aza-spiro [3.4] octan-1-one 4a and 7 $\beta$-iodo-2-(4'-methoxyphenyl)-3phenyl-5-thia-2-aza-spiro [3.4] octan-1-one 5a. The absence of singlet for methylthio group and appearance of multiplet for $-\mathrm{CH}_{2}$ groups confirmed that the substrate $\mathbf{3}$ has cyclized to give $\mathrm{C}-3$ spiro- $\beta$-lactams $\mathbf{4}$ and 5 (table 2).

Table 1. Synthesis of cis-3-allyl-3-methylthioazetidin-2ones 3 .

\begin{tabular}{lcccc}
\hline Entry & $\mathrm{R}^{1}$ & \multicolumn{1}{c}{$\mathrm{R}^{2}$} & Products & $\%$ yield $^{\mathrm{a}}$ \\
\hline 1 & $\mathrm{C}_{6} \mathrm{H}_{5}$ & $p-\mathrm{C}_{6} \mathrm{H}_{5} \mathrm{OMe}$ & $\mathbf{3 a}$ & 75 \\
2 & $\mathrm{C}_{6} \mathrm{H}_{5}$ & $\mathrm{C}_{6} \mathrm{H}_{5}$ & $\mathbf{3 b}$ & 76 \\
3 & $p-\mathrm{C}_{6} \mathrm{H}_{5} \mathrm{OMe}$ & $p-\mathrm{C}_{6} \mathrm{H}_{5} \mathrm{OMe}$ & $\mathbf{3 c}$ & 72 \\
4 & $\mathrm{C}_{6} \mathrm{H}_{5}$ & $p-\mathrm{C}_{6} \mathrm{H}_{5} \mathrm{Me}$ & $\mathbf{3 d}$ & 74 \\
\hline
\end{tabular}

${ }^{\text {a }}$ Yields quoted are for the isolated products

Table 2. $\quad$ C-3 Spiro- $\beta$-lactams 4 and 5.

\begin{tabular}{lccc}
\hline Entry & $\mathrm{R}^{1}$ & $\mathrm{R}^{2}$ & Product $\left(\%\right.$ yield $\left.^{\mathrm{a}}\right)$ \\
\hline 1 & $\mathrm{C}_{6} \mathrm{H}_{5}$ & $p-\mathrm{C}_{6} \mathrm{H}_{4} \mathrm{OMe}$ & $\mathbf{4 a} \boldsymbol{\&} \mathbf{5 b}(83)$ \\
2 & $\mathrm{C}_{6} \mathrm{H}_{5}$ & $\mathrm{C}_{6} \mathrm{H}_{5}$ & $\mathbf{4 b} \boldsymbol{\&} \mathbf{5 b}(71)$ \\
3 & $p-\mathrm{C}_{6} \mathrm{H}_{4} \mathrm{OMe}$ & $p-\mathrm{C}_{6} \mathrm{H}_{4} \mathrm{OMe}$ & $\mathbf{4 c} \boldsymbol{\mathbf { 5 }} \mathbf{c}(80)$ \\
4 & $\mathrm{C}_{6} \mathrm{H}_{5}$ & $p-\mathrm{C}_{6} \mathrm{H}_{4} \mathrm{Me}$ & $\mathbf{4 d} \boldsymbol{\&} \mathbf{5 d}(69)$ \\
\hline
\end{tabular}

a Yields quoted are for the isolated products
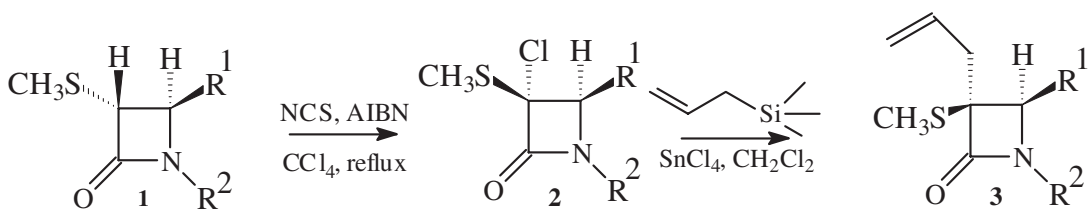

Scheme 1. Synthesis of cis-3-allyl-3-methylthio- $\beta$-lactams 3.
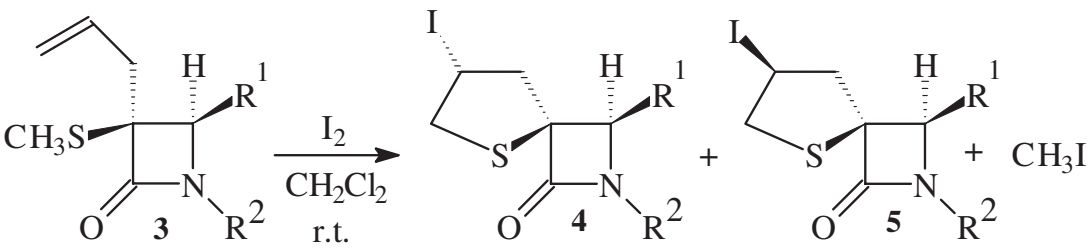

Scheme 2. Synthesis of spiro- $\beta$-lactams 4 and 5. 


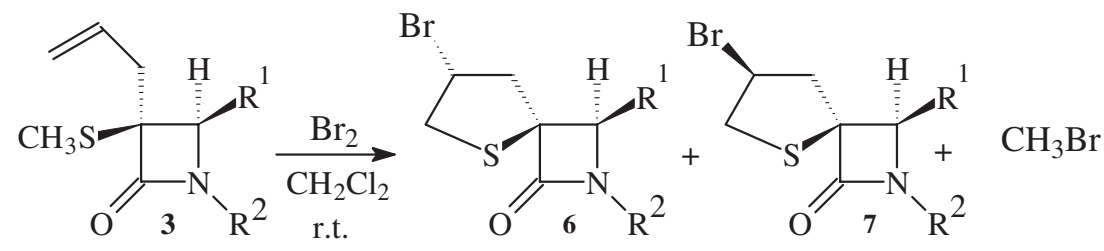

Scheme 3. Synthesis of spiro- $\beta$-lactams 6 and 7.

Table 3. C-3 Spiro- $\beta$-lactams 6 and 7.

\begin{tabular}{llll}
\hline Entry & $\mathrm{R}^{1}$ & $\mathrm{R}^{2}$ & Product $\left(\%\right.$ yield $\left.^{\mathrm{a}}\right)$ \\
\hline 1 & $\mathrm{C}_{6} \mathrm{H}_{5}$ & $p-\mathrm{C}_{6} \mathrm{H}_{4} \mathrm{OMe}$ & $\mathbf{6 a}(28), \mathbf{7 a}(30)$ \\
2 & $\mathrm{C}_{6} \mathrm{H}_{5}$ & $\mathrm{C}_{6} \mathrm{H}_{5}$ & $\mathbf{6 b} \mathbf{\&} \mathbf{7 b}(67)$
\end{tabular}

${ }^{a}$ Yields quoted are for the isolated products.
The structures of spiro- $\beta$-lactams $\mathbf{4}$ and $\mathbf{5}$ were established on the basis of IR, ${ }^{1} \mathrm{H}$ NMR and ${ }^{13} \mathrm{C}$ NMR. The stereochemical assignment at $\mathrm{C}-3$ spiro junction of spiro- $\beta$-lactams $\mathbf{4}$ and $\mathbf{5}$ was made on the basis of correlation of ${ }^{1} \mathrm{H}$ NMR and ${ }^{13} \mathrm{C}$ NMR data of these $\beta$-lactams with that of spiro- $\beta$-lactams prepared from

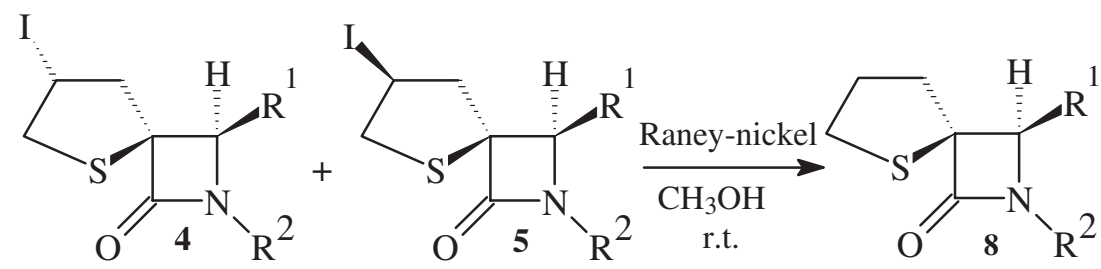

Scheme 4. Dehalogenation studies of spiro- $\beta$-lactams.<smiles>[Y4]C1CC1[R]N1C(=O)C(CC=C)(SC)C1[Y]</smiles>

3<smiles>[Y4]C=CCC1(SC)C(=O)N([R])[C@H]1[R]</smiles>
$(\pi$-Complex)

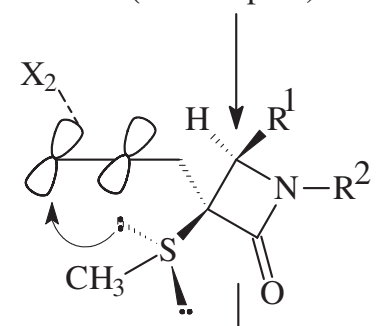

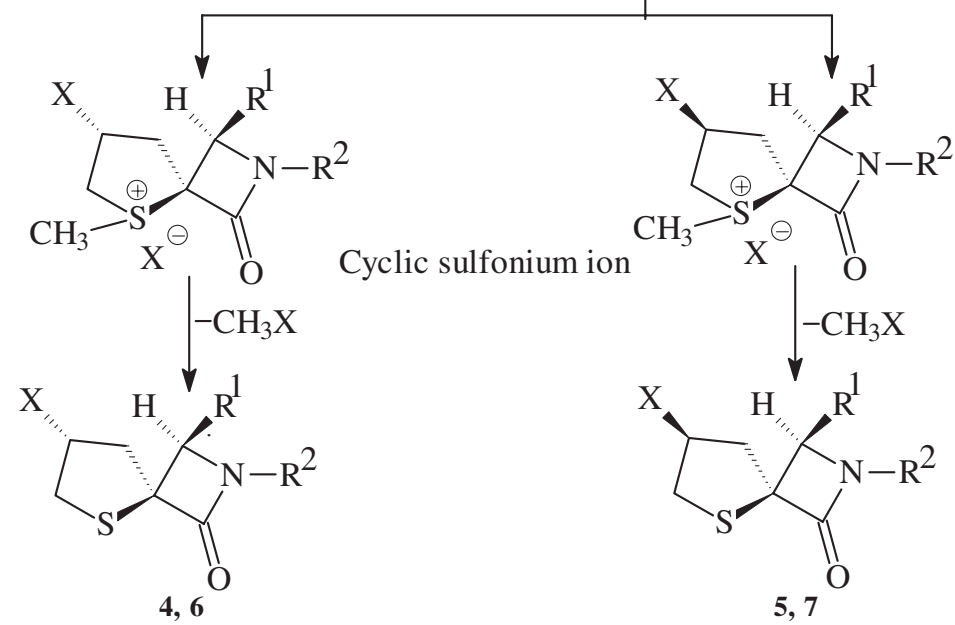

Scheme 5. Plausible mechanism for formation of spiro- $\beta$-lactams 4 -7. 
3-allyl-3-benzylthio- $\beta$-lactams, whose stereochemistry has been confirmed by X-ray crystallographic studies (scheme 3). ${ }^{16}$

These 3-allyl-3-methylthio- $\beta$-lactams 3 were then subjected to bromine-mediated intrasulfenyl cyclization reaction. Initially, 3a was treated with 1 equiv. of bromine in dry dichloromethane at room temperature. The progress of the reaction was monitored by TLC. The TLC of the reaction mixture showed the appearance of two spots having $\mathrm{R}_{\mathrm{f}}$ lower than the spot for starting substrate. The products, after work up were separated by column chromatography and subsequently identified using spectroscopic studies. The fast moving product was identified as $7 \alpha$-bromo2-(4'-methoxyphenyl)-3-phenyl-5-thia-2-aza-spiro[3.4] octan-1-one $6 \mathbf{a}$ and the slow moving product was identified as 7 $\beta$-bromo-2-(4'-methoxyphenyl)-3-phenyl-5thia-2-aza-spiro[3.4] octan-1-one 7a.

The reaction was found to be successful with $\mathbf{3 d}$ also and the results are summarized in table 3 .

Thus, it is clear that halocyclization reaction of $\beta$-lactams of type 3(a-d) led to the formation of both $\alpha$ - and $\beta$-epimers and in same isomeric ratio as given by 3-benzylthio substrate. The isomeric ratio was determined from NMR of crude sample. Further, the substrate cyclize to give five-membered rings, exclusively. The regiochemical selectivity of alkenyl ring closure reflect the thermodynamic differences between the two possible $\beta$-halosulfide region adducts.

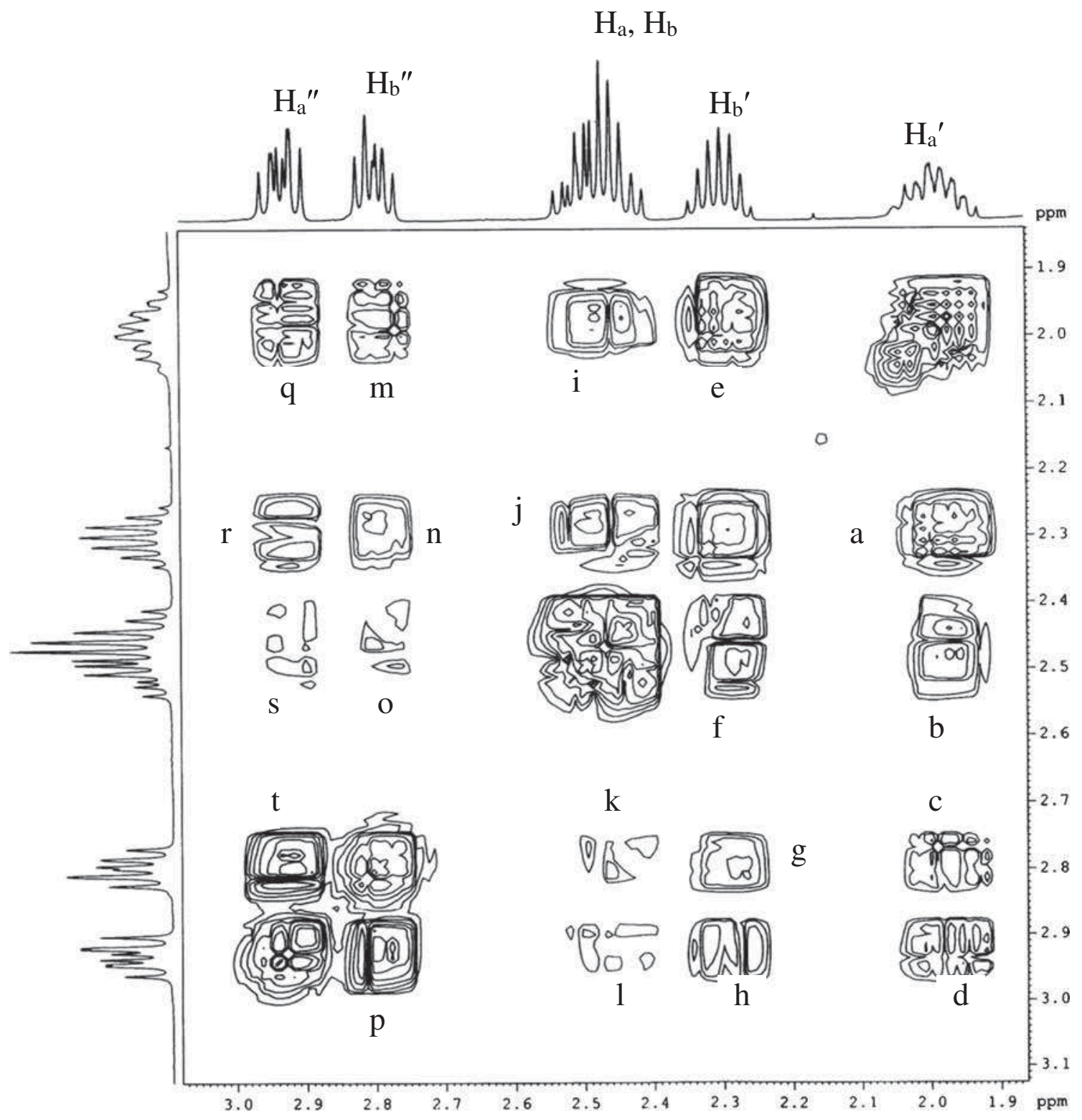

Figure 1. Homonuclear ${ }^{1} \mathrm{H}-{ }^{1} \mathrm{H}$ correlation spectrum, COSY for compound $8 \mathbf{a}$. 
The formation of five membered ring can be rationalized on the basis of the plausible mechanism, which is presented below (scheme 4):

The reaction first involves the coordination by halogen to the double bond of $\beta$-lactam 3(a-d) to produce a $\pi$-complex. Subsequently, nucleophilic addition of the sulfide center to the halogen-olefin complex gives the cyclic sulfonium ion, which undergoes further dealkylation by halide ion to produce methyl halide and five membered ring spiro[3.4] $\beta$-lactams 4-7.

These $\mathrm{C}-3$ spiro- $\beta$-lactams $(4,5)$ were subjected to dehalogenation reaction. Initially, mixture of $\mathbf{4 a}$ and 5a was treated with Raney-nickel in methanol at room temperature. The progress of the reaction was monitored by TLC. After $30 \mathrm{~min}$, the TLC profile of the reaction mixture showed one spot having $\mathrm{R}_{\mathrm{f}}$ lower than the spot for starting mixture of substrates. The residue, after work up, was subjected to purification by column chromatography. The resulting product was identified as 2-(4'-methoxyphenyl)-3-phenyl-5thia-2-aza-spiro[3.4]octan-1-one 8a (79\%) on the basis of spectral data. In DEPT 135 of $\mathbf{8 a}$, three resonances at $\delta 30.38,34.08$ and $39.69 \mathrm{ppm}$ with negative amplitude confirmed the presence of three methylenes (scheme 5).
Further, the structure of 5 -membered ring spiro- $\beta$ lactams was corroborated from the NMR studies using correlation spectroscopy such as proton-proton COSY $\left({ }^{1} \mathrm{H}-{ }^{1} \mathrm{H} \mathrm{COSY}\right)$ and heteronuclear single quantum correlation (HSQC) (figure 1).

The proton-proton COSY spectrum of $8 \mathrm{a}$ shows that proton, $\mathrm{H}_{\mathrm{a}}^{\prime}$ appearing at $\delta 1.96-2.03 \mathrm{ppm}$ correlates (a and e, b and i, c and $\mathrm{m}, \mathrm{d}$ and q) with geminal, $\mathrm{H}_{\mathrm{b}}^{\prime}$ and neighbouring protons, $\mathrm{H}_{\mathrm{a}}, \mathrm{H}_{\mathrm{b}}, \mathrm{H}_{\mathrm{b}}^{\prime \prime}$ and $\mathrm{H}_{\mathrm{a}}^{\prime \prime}$. Similarly, $\mathrm{H}_{\mathrm{b}}^{\prime}$ correlates (e and $\mathrm{a}, \mathrm{f}$ and $\mathrm{j}, \mathrm{g}$ and $\mathrm{n}, \mathrm{h}$ and $\mathrm{r}$ ) with geminal $\mathrm{H}_{\mathrm{a}}^{\prime}$ and neighbouring protons, $\mathrm{H}_{\mathrm{a}}, \mathrm{H}_{\mathrm{b}}, \mathrm{H}_{\mathrm{b}}^{\prime \prime}$ and $\mathrm{H}_{\mathrm{a}}^{\prime \prime}$. Further, protons $\mathrm{H}_{\mathrm{a}}$ and $\mathrm{H}_{\mathrm{b}}$ appearing at $\delta 2.41-2.53 \mathrm{ppm}$ correlate (i and b, j and $\mathrm{f}, \mathrm{k}$ and $\mathrm{o}, \mathrm{l}$ and $\mathrm{s}$ ) with $\mathrm{H}_{\mathrm{a}}^{\prime}$, $\mathrm{H}_{\mathrm{b}}^{\prime}, \mathrm{H}_{\mathrm{b}}^{\prime \prime}$ and $\mathrm{H}_{\mathrm{a}}^{\prime \prime}$. The proton, $\mathrm{H}_{\mathrm{b}}^{\prime \prime}$ appearing at $\delta 2.76-$ $2.82 \mathrm{ppm}$ correlates ( $\mathrm{m}$ and $\mathrm{c}, \mathrm{n}$ and $\mathrm{g}, \mathrm{o}$ and $\mathrm{k}, \mathrm{p}$ and $\mathrm{t}$ ) with neighbouring protons, $\mathrm{H}_{\mathrm{a}}^{\prime}, \mathrm{H}_{\mathrm{b}}^{\prime}, \mathrm{H}_{\mathrm{a}}, \mathrm{H}_{\mathrm{b}}$ and geminal proton $\mathrm{H}_{\mathrm{a}}^{\prime \prime}$. Similarly, $\mathrm{H}_{\mathrm{a}}^{\prime \prime}$, appearing at $\delta 2.90-2.96 \mathrm{ppm}$ correlates ( $\mathrm{q}$ and $\mathrm{d}, \mathrm{r}$ and $\mathrm{h}, \mathrm{s}$ and $\mathrm{l}, \mathrm{t}$ and $\mathrm{p}$ ) with neighbouring protons, $\mathrm{H}_{\mathrm{a}}^{\prime}, \mathrm{H}_{\mathrm{b}}^{\prime}, \mathrm{H}_{\mathrm{a}}, \mathrm{H}_{\mathrm{b}}$ and geminal proton $\mathrm{H}_{\mathrm{b}}^{\prime \prime}$ (figure 2).

The HSQC of 8a shows that resonance at $\delta$ $30.36 \mathrm{ppm}$ on carbon spectrum corresponds to $\mathrm{H}_{\mathrm{a}}^{\prime \prime}$, $\mathrm{H}_{\mathrm{b}}^{\prime \prime}$ protons ( $\mathrm{a}$ and $\mathrm{b}$ ). The resonance at $\delta 39.67$ and $34.06 \mathrm{ppm}$ corresponds to $\mathrm{H}_{\mathrm{a}}, \mathrm{H}_{\mathrm{b}}$ protons (c) and $\mathrm{H}_{\mathrm{a}}^{\prime}$, $\mathrm{H}_{\mathrm{b}}^{\prime}$ protons (e and d) respectively. Surprisingly, $\mathrm{H}_{\mathrm{a}}$ and

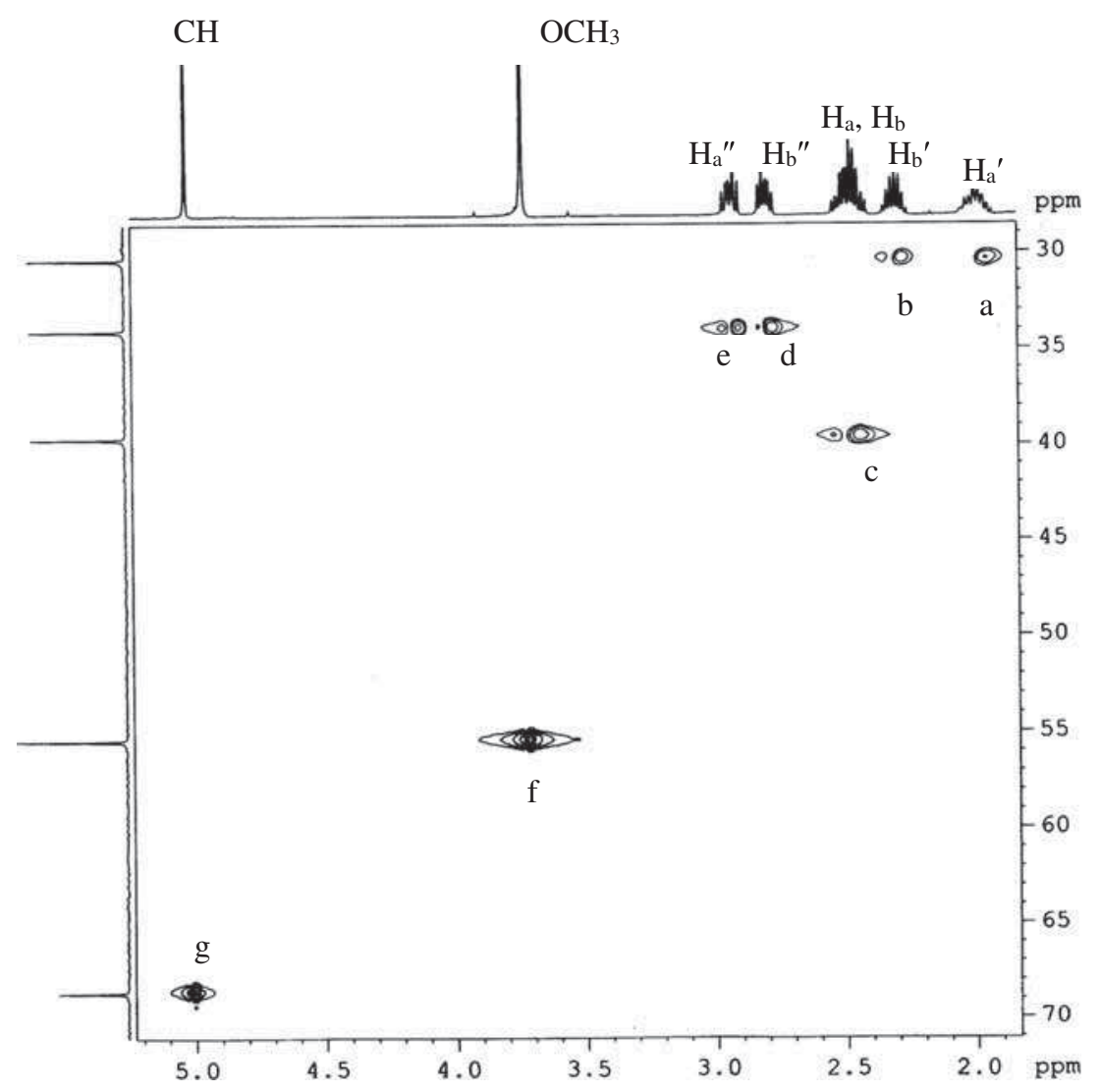

Figure 2. Heteronuclear ${ }^{1} \mathrm{H}-{ }^{13} \mathrm{C}$ correlation spectrum, HSQC for $8 \mathbf{8}$. 
$\mathrm{H}_{\mathrm{b}}$ protons appear in the upfield region, but the carbon to which they are attached appears in the downfield region. The possible reason could be that secondary carbon attached to the sulfur is in the cone of sulfur and the protons attached to it are out of the deshielded region of sulfur. The carbon peak at $\delta 55.44$ and 68.68 ppm corresponds to methoxy protons (f) attached to aryl ring at 4-position and $\mathrm{CH}(\mathrm{g})$, respectively.

The dehalogenation reaction was found to be successful with 4(b-d) and 5(b-d) and the results are summarized in table 4 . The structures of azetidin-2-ones $\mathbf{8}(\mathbf{a}-\mathbf{d})$ were established on the basis of their spectral data such as IR, ${ }^{1} \mathrm{H}$ NMR, ${ }^{13} \mathrm{C}$ NMR and DEPT 135.

The C-3 Spiro- $\beta$-lactams 8(a-d) were furthur subjected to oxidation reaction to form sulfoxide derivatives. Initial studies were carried out by treating 8a with $m$-chloroperbenzoic acid in dichloromethane at $0^{\circ} \mathrm{C}$. The reaction mixture was stirred and monitored by TLC. After $15 \mathrm{~min}$, the TLC profile of the reaction mixture showed the appearance of two spots, having $R_{f}$ lower than the starting substrate. After usual work up, the products were separated by column chromatography (scheme 6).

The first eluting product was identified as 2-(4'methoxyphenyl)-5-oxo-3-phenyl-5 $\lambda^{4}$-thia-2-aza-spiro [3.4]octan-1-one 9a (39\%) and the slow moving product was identified as 10a (50\%) as a crystalline solid on the basis of spectral data.

In DEPT 135 of $9 \mathbf{a}$, three resonances at $\delta 22.78$, 28.51 and $51.80 \mathrm{ppm}$ and 10a $\delta 23.45,30.82$ and 52.65 with negative amplitude confirmed the presence of three methylenes. Further, the assignment of the protons was done from the NMR analysis using correlation spectroscopy such as proton-proton COSY and heteronuclear single quantum correlation (HSQC). The reaction

Table 4. C-3 Spiro- $\beta$-lactams 8(a-d).

\begin{tabular}{lccc}
\hline Entry & $\mathrm{R}^{1}$ & $\mathrm{R}^{2}$ & Product (\% yield) \\
\hline 1 & $\mathrm{C}_{6} \mathrm{H}_{5}$ & $p-\mathrm{C}_{6} \mathrm{H}_{4} \mathrm{OMe}$ & $\mathbf{8 a}(79)$ \\
2 & $\mathrm{C}_{6} \mathrm{H}_{5}$ & $\mathrm{C}_{6} \mathrm{H}_{5}$ & $\mathbf{8 b}(77)$ \\
3 & $p-\mathrm{C}_{6} \mathrm{H}_{4} \mathrm{OMe}$ & $p-\mathrm{C}_{6} \mathrm{H}_{4} \mathrm{OMe}$ & $\mathbf{8 c}(73)$ \\
4 & $\mathrm{C}_{6} \mathrm{H}_{5}$ & $p-\mathrm{C}_{6} \mathrm{H}_{4} \mathrm{Me}$ & $\mathbf{8 d}(70)$ \\
\hline
\end{tabular}

${ }^{\text {a }}$ Yields quoted are for the isolated products. was found to be general for several substrates and results are summarized in table 5 . The stereochemistry was established through single crystal X-ray crystallographic studies of compound 9a as shown in ORTEP presentation given below (figure 3):

C-3 Spiro- $\beta$-lactams 8(a-d) were also studied for sulfone derivatives. Initially, 8a was treated with 2 equiv. of $m$-chloroperbenzoic acid in dichloromethane at room temperature. The progress of the reaction was monitored by TLC. After TLC indicated the disappearance of the spot corresponding to the starting substrate and appearance of the spot having $R_{\mathrm{f}}$ lower than the spot of starting substrate, the reaction mixture was poured into $5 \%$ aqueous $\mathrm{NaHSO}_{3}$ solution. After usual work up, the crude product was purified by column chromatography (scheme 7).

The product obtained was identified as 2-(4'methoxyphenyl)-3-(4'-methoxyphenyl)-5,5-dioxo-5 $\lambda^{6}$ thia-2-aza-spiro[3.4]octan-1-one, 11a on the basis of

Table 5. Sulfoxide derivatives $\mathbf{9}(\mathbf{a}-\mathbf{d})$ and $\mathbf{1 0}(\mathbf{a}-\mathbf{d})$.

\begin{tabular}{lccc}
\hline Entry & $\mathrm{R}^{1}$ & $\mathrm{R}^{2}$ & Product (\% yield) \\
\hline 1 & $\mathrm{C}_{6} \mathrm{H}_{5}$ & $p-\mathrm{C}_{6} \mathrm{H}_{4} \mathrm{OMe}$ & $\mathbf{9 a}(39) \mathbf{1 0 a}(50)$ \\
2 & $\mathrm{C}_{6} \mathrm{H}_{5}$ & $\mathrm{C}_{6} \mathrm{H}_{5}$ & $\mathbf{9 b}(41) \mathbf{1 0 b}(47)$ \\
3 & $p-\mathrm{C}_{6} \mathrm{H}_{4} \mathrm{OMe}$ & $p-\mathrm{C}_{6} \mathrm{H}_{4} \mathrm{OMe}$ & $\mathbf{9 c}(47) \mathbf{1 0 c}(42)$ \\
4 & $\mathrm{C}_{6} \mathrm{H}_{5}$ & $p-\mathrm{C}_{6} \mathrm{H}_{4} \mathrm{Me}$ & $\mathbf{9 d}(33) \mathbf{1 0 d}(40)$ \\
\hline
\end{tabular}

${ }^{a}$ Yields quoted are for the isolated products.

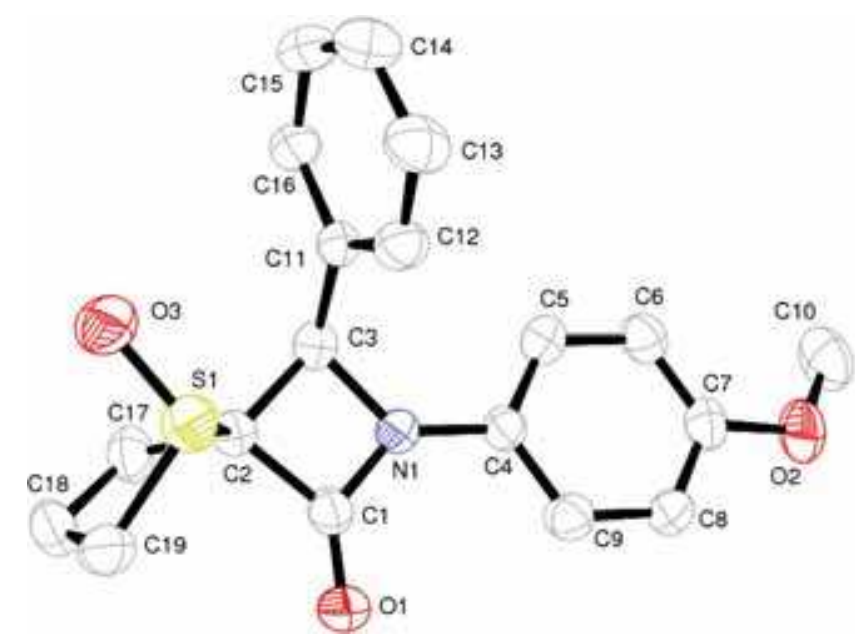

Figure 3. An ORTEP diagram of compound 9a.

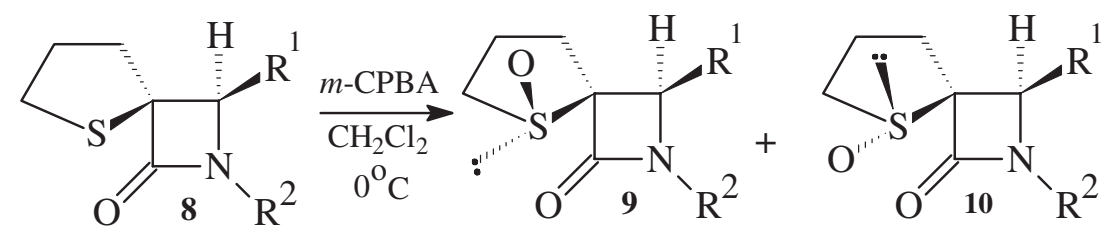

Scheme 6. Synthesis of spirosulfoxide- $\beta$-lactams 9 and $\mathbf{1 0}$. 


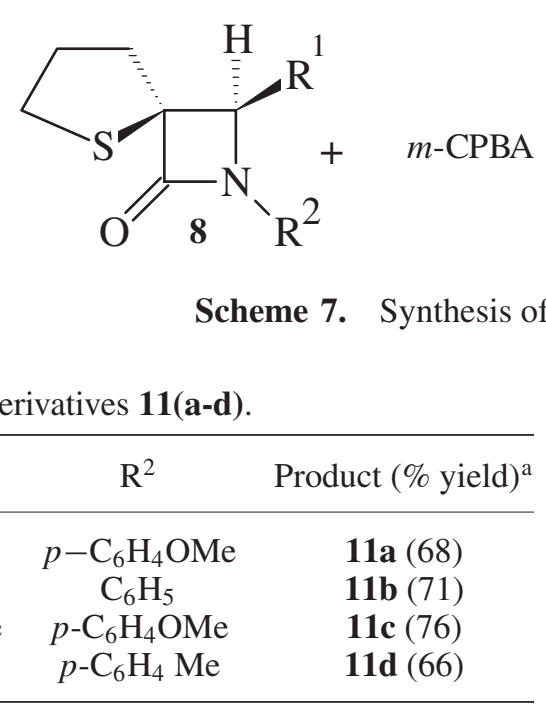

${ }^{\text {a }}$ Yields quoted are for the isolated products.

spectral data. The reaction was found to be successful with substrates $8(\mathrm{~b}-\mathrm{d})$ and the results are summarized in table 6 . The structures of the products $11(\mathrm{~b}-\mathrm{d})$ were established on the basis of their spectral data such as IR, ${ }^{1} \mathrm{H}$ NMR, ${ }^{13} \mathrm{C}$ NMR and DEPT 135.

\section{Conclusion}

In conclusion, we have described the synthesis of $\mathrm{C}$ 3 spiro- $\beta$-lactams by using $c i s$-3-allyl-3-methylthio- $\beta$ lactams as the substrate. This proved to be equally efficient compound for halocyclization although it provides same isomeric ratio of products as given by $\mathrm{cis}$ 3 -allyl-3-benzylthio- $\beta$-lactams. Also these C-3 spiro$\beta$-lactams after dehalogenation are converted into their sulfoxide and sulfone derivatives using $m$-CPBA acid as an oxidizing agent. Furthur elaboration of this work to potentially useful building blocks is underway in our lab.

\section{Supplementary Information}

All additional information pertaining to characterization of the representative compounds $(\mathbf{4 d}, \mathbf{5 d}, \mathbf{8 c}, \mathbf{9 b}$, 10b, 11b) using ${ }^{1} \mathrm{H}$ NMR and ${ }^{13} \mathrm{C}$ NMR technique (Figures S1-S5) are given in the supporting information. Supplementary Information is available at www. ias.ac.in/chemsci.

\section{Acknowledgement}

We gratefully acknowledge the financial support for this work from Council of Scientific and Industrial Research (CSIR), New Delhi, India.

\section{References}

1. (a) G I Georg (ed.) 1993 In The Organic Chemistry of $\beta$-lactams (New York: VCH); (b) Nau R and Eiffert A 2002 Clin. Microb. Rev. 1595

2. (a) Palomo C 1990 In Recent Progress in the Chemical Synthesis of Antibiotics G Luckacs and M Ohno M (eds.) (Berlin: Springer); (b) Palomo C, Aizpurua J M, Ganboa I and Oiarbide M 2000 Pure Appl. Chem. 72 1763; (c) Palomo C, Aizpurua J M, Ganboa I and Oiarbide M 2001 Synlett 1813

3. Skiles J W and McNeil D 1990 Tetrahedron Lett. 31 7277

4. Sheehan J C, Chacko E, Lo Y S, Ponzi D R and Sato E 1978 J. Org. Chem. 424856

5. Wu G and Tormos W 1997 J. Org. Chem. 626412 and references cited therein

6. Alonso E, Lopez-Ortiz F, del Pozo C, Peralta E, Macias A and Gonzalez J 2001 J. Org. Chem. 666333

7. Alonso E, del Pozo C and Gonzalez J 2002 Synlett. 69

8. Selected recent references. (a) Strauss A and Otto H H 1997 Helv. Chim. Acta 80 1823; (b) Anklam S and Liebscher J 1998 Tetrahedron 54 6369; (c) Dalla-Croce P and La Rosa C 1990 Tetrahedron: Asymmetry 10 1193; (d) Barba V, Hernandez C, Rojas-Lima S, Farfan N and Santillan R 1999 Can. J. Chem. 77 2025; (e) Papillon J P B and Taylor R J K 2000 Org. Lett. 21987

9. Singh G S, D'hooge M and Kimpe N 2011 Tetrahedron 671989

10. Santos B S, Nunes C C, Pias A C C and e Melo P T 2012 Tetrahedron 683729

11. Cremonesi G, Croce P D, Forni A and Rosa C L 2013 Tetrahedron 691175

12. Selected recent reviews. (a) Yamamoto Y, Asao N and Tsukuda N 1998 In Advances in Asymmetric synthesis Vol. 3 A H Hassner (Ed.) Asymmetric Synthesis of $\beta$ Lactam Derivatives via Conjugate Addition of metal Amides (Stamford, CT: JAI Press); (b) Muller T E and Beller M 1998 Chem. Rev. 98 675; (c) Doyle M P 1998 Pure Appl. Chem. 70 1123; (d) Benaglia M, Cinquini M and Cozzi F 2000 Eur. J. Org. Chem. 563; (e) Dirat O, Koulovsky C, Manduit M and Langlois Y 2000 Pure Appl. Chem. 72 1721; (f) Kawabata T 2000 Rev. Heteroatom Chem. 33; (g) For solid phase and combinatorial synthesis, see. Mata E G 1999 Curr. Pharm. Des. 5955

13. Palomo C, Aizpurua J M, Ganboa I and Oiarbide M 1999 Eur. J. Org. Chem. 3223 and references cited therein

14. Bari S S, Reshma, Bhalla A and Hundal G 2009 Tetrahedron $\mathbf{6 5} 10060$

15. Bhalla A, Venugopalan P and Bari S S 2006 Eur. J. Org. Chem. 4943

16. Arora R, Venugopalan P and Bari S S 2010 J. Chem. Sci. 122125 